\title{
Multichannel satellite image application for water surface objects identification
}

\author{
Natalia Panasenko ${ }^{1}$, Marina Ganzhur ${ }^{1, *}$, Alexey Ganzhur ${ }^{1}$, and Vladimir Fathi ${ }^{1}$ \\ ${ }^{1}$ Don State Technical University, 1, Gagarin sq., 344000, Rostov on Don, Russia
}

\begin{abstract}
The paper is devoted to the analysis of methods of adoption of satellite observation data in order to identify the required information used in the development and verification of mathematical models of hydrodynamics and biological kinetics of shallow water reservoirs. For the information accumulation, we consider the use of remote sensing data. The aim of the paper is to identify the best implementation method for software tools in order to improve the quality of assimilation of date of satellite sensing of the Earth relating to hydrobiological processes in a shallow water reservoir.
\end{abstract}

\section{Introduction}

A huge number of Russian and foreign scientists are engaged in mathematical modeling of processes of hydrodynamics and biological kinetics of problems in aquatic ecosystems. Currently, there are more than 4,000 ecological models for various purposes and complexity levels. A huge contribution in the field of creating mathematical models, developing diagnostic methods and predicting changes in aquatic ecosystems was made through fundamental works by such authors as Lotka A.J. [1], Volterra V. [2], Marchuk G.I. [3], Ozmidov R.V. [4], Gause G.F., [5] where the issues of interspecies interaction were considered. Hutchinson G.E. studied the flora and fauna of lakes. Mitscherlich E. A. discovered the law of influence of growth factors and presented a new look at the science of plants [6]. Vinberg G.G. [7] conducted research related to the processes of nutrition and growth of aquatic organisms. Vorovich I.I. and Gorstko A.B. [8] developed schemes for the rational use of water resources. Odum H.T. explained ecological structures by energy systems [9]. Abakumov A.I. [10] is actively involved in the development of methods for optimizing modeling of aquatic ecosystems. Jorgensen S.E., Meyer H., Firiis M. [11] studied the issues of limnology and ecological modeling. Vollenweider R.A. studied the eutrophication of lakes. And despite their diversity, the models are aimed at achieving one main goal - an adequate description of the aquatic ecosystems functioning.

Currently, for the effective use of these and many other models for the purpose of analysis and forecasting, there is a significant problem of equipping them with real input data. As a source of such input data in modern conditions, it is advisable to use information from artificial Earth satellites, as well as from unmanned aerial vehicles and other remote sources of geophysical information [12]. They allow obtaining the main parameters of

\footnotetext{
*Corresponding author: natalija93_93@mail.ru
} 
complex natural systems, which are coastal and marine systems. These include cloudiness parameters, surface topography, velocity of movement of the aquatic environment, distribution of temperatures and salinity in the near-surface layer, wind speed and direction, and chlorophyll concentration. The most important challenge that needs to be addressed is the development of methods and tools for the use of heterogeneous satellite data. In this regard, the purpose of this paper is to describe methods of recognition and adoption of date of space shooting of phytoplankton algae "blooming".

\section{Challenges consideration}

In the last decade, the great importance for Russia is studying its territory using satellite methods. The main areas of application of the Earth remote sensing (ERS) are obtaining information on the state of the environment and land use, studying plant communities, assessing the yield of agricultural crops and consequences of natural disasters [13].

To date, a huge number of satellites, both Russian and foreign, have been launched (Resource-P, WorldView, Landsat, KompSat, SPOT, Canopus-V, GeoEye, Radarsat, Resource-F and many others), which cover most of the planet. However, obtaining "cloudless" images and images of various scales is a big problem. In practice, there is a need to use a set from different satellites. However, what are we considering?

Phytoplankton is the basis of the trophic pyramid of biogeocenosis of a shallow water reservoir, the main link in the "phytoplankton-zooplankton-fish" food chain. With the flow of rivers, nutrients being compounds of nitrogen, silicon and phosphorus enter water reservoirs, causing a sharp increase in the phytoplankton population called "blooming" [14].

The problem of intensive "blooming" of water is very pressing for a wide variety of water areas. The "blooming" of water is considered to be the massive development of one or 2-3 planktonic species, accompanied by a significant deterioration in water quality. Intense and often harmful algae blooming has increased dramatically in the last 20-30 years and have spread to both fresh and marine water reservoirs. According to hydrobiologists, this is caused by a whole complex of factors, such as climate change, entry of a large amount of various mineral and organic substances into the water which is associated with the intensification of human economic activity [15].

When analyzing information from satellites, areas of intense blooming appear very clear in the images of the visible range. In Fig. 1, we present color-synthesized images from WorldView [16] and Resource-P [17] satellites obtained on September 10, 2019 over the water area of the Azov Sea. Large-scale manifestations of a cyclonic swirl are a clear example of how biogenic pollutants caused by intense blooming of cyanobacteria spread under the influence of dynamic and circulating processes. 


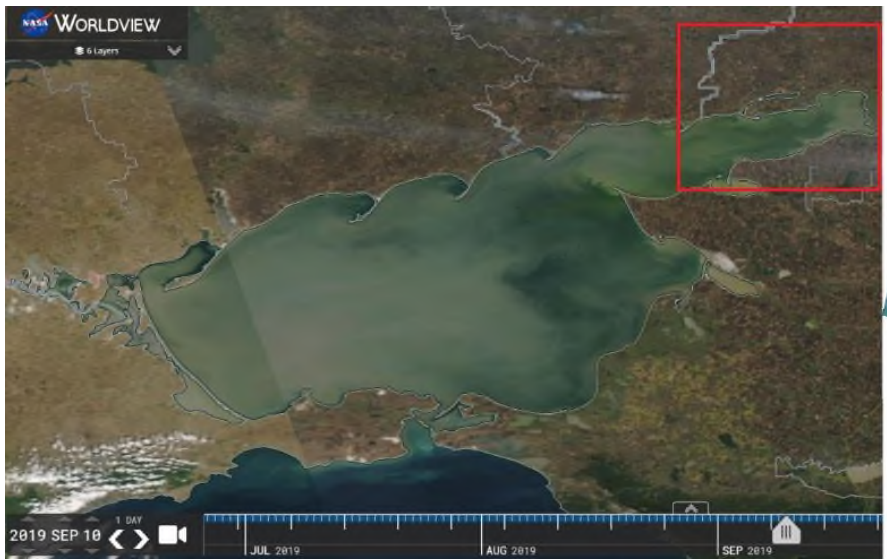

a)

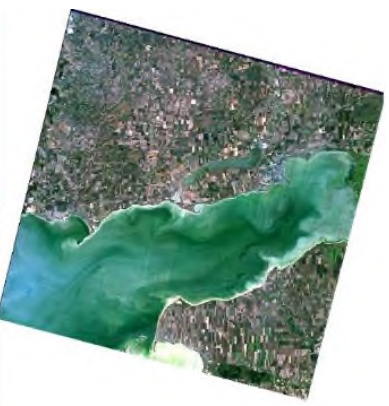

b)

Fig. 1. Propagation of impurities: a) WorldView satellite; b) Resource-P satellite

The contours accentuation plays an important role in image analysis and pattern recognition when solving computer vision problems. Being points of difference in brightness values in a grayscale image, they are important image features. These sharp transition points indicate the location of objects in the images, allowing reduction of the data amount while retaining important information about the shape of objects in the scene.

For the further analysis of the impurities propagation, we need to consider various algorithms that will allow us to determine the contours of images. The image contour is meant by us as a spatially extended discontinuity, drop or abrupt change in brightness values. Currently, there are a large number of different algorithms, but only a few of them are widespread due to their versatility. In this paper, the most popular algorithms will be considered: Sobel operator, Kirsch operator, Laplace operator and method based on Local Binary Patterns (LBP). In order to test these methods, a test image (Fig. 2) from the WorldView satellite [16] will be used, obtained on April 4, 2020 over the water area of the Azov Sea.

Let us select the required area for work, build a grid and define the area manually for the recognition comparison. This information will allow us to further consider the effectiveness of the selected methods (Fig. 3).

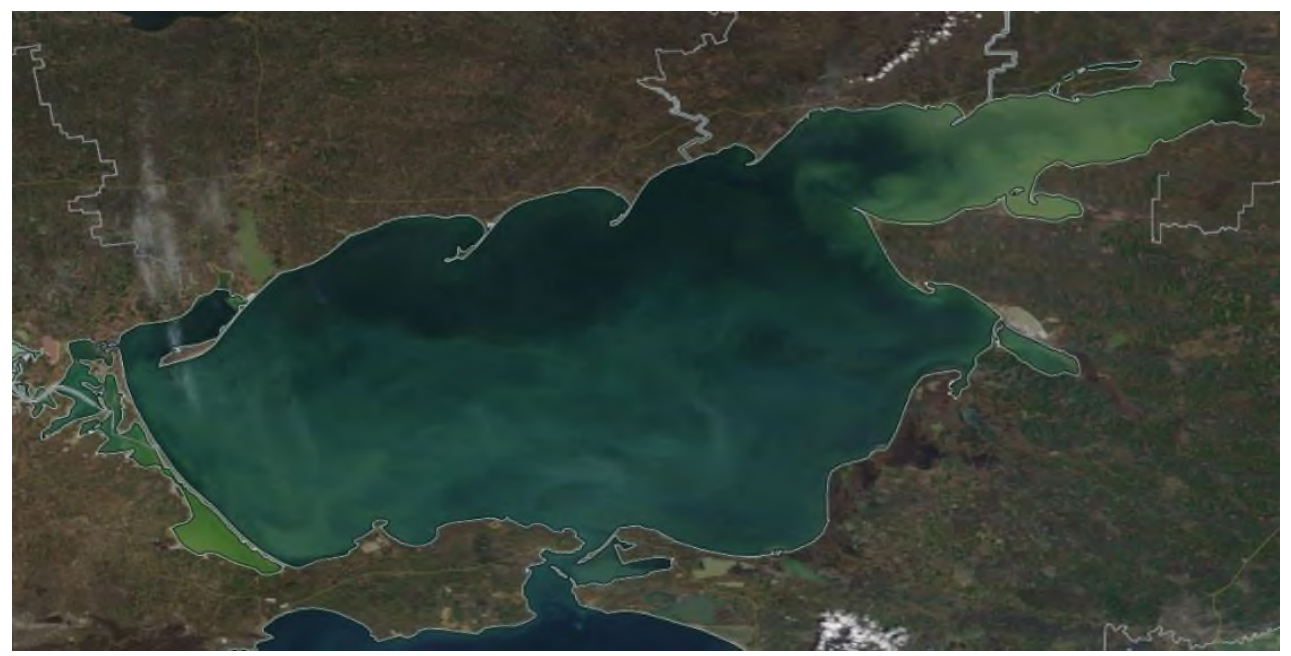


Fig. 2. Source image for contours detection

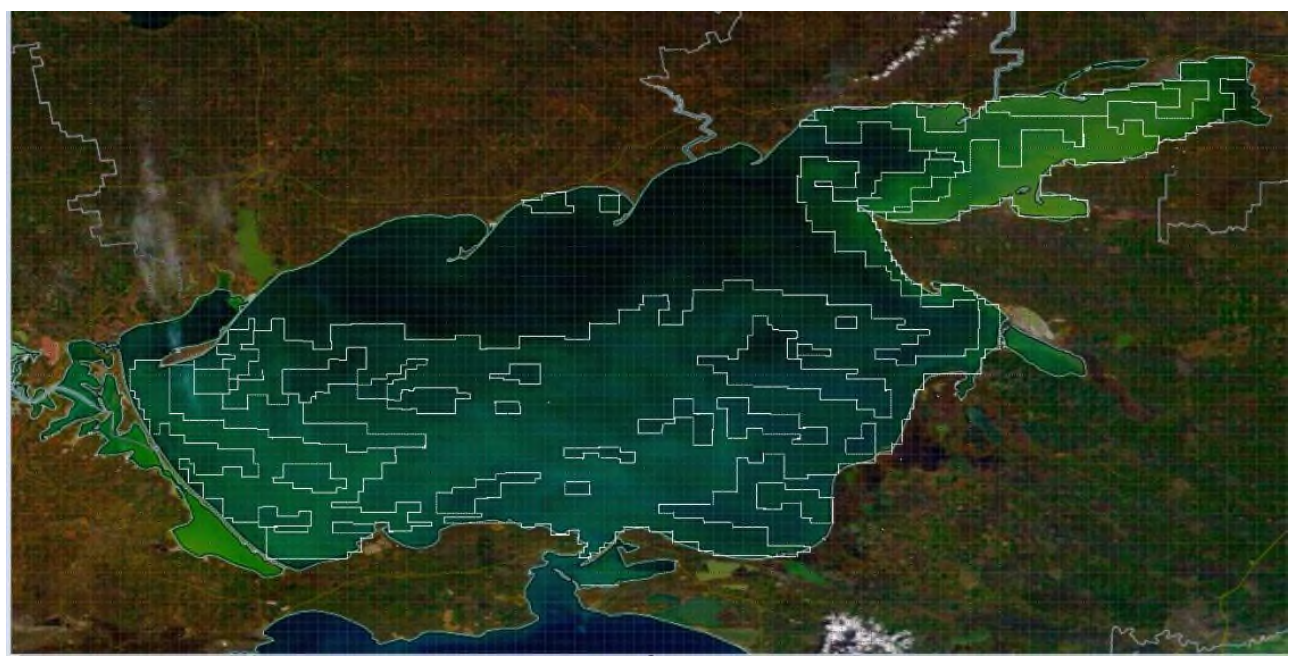

Fig. 3. Detection of the recognized area

We start our consideration with the contours accentuation algorithm by the Sobel method $[18,19]$. It was introduced in 1970. This operator consists of two matrices (1) and is based on the central difference concept. However, the weight of center pixels is doubled. The Sobel operator uses weighting factor 2 for middle items. This increased value is used for reducing the smoothing effect by giving more weight to the midpoints.

$$
G_{x}=\left[\begin{array}{lll}
-1 & 0 & +1 \\
-2 & 0 & +2 \\
-1 & 0 & +1
\end{array}\right], G_{y}=\left[\begin{array}{ccc}
-1 & -2 & -1 \\
0 & 0 & 0 \\
+1 & +2 & +1
\end{array}\right]
$$

To calculate the gradient value, these components should be used together:

$$
G=\sqrt{G_{x}^{2}-G_{y}^{2}}
$$

As a result of the software implementation, we get Figure 4.

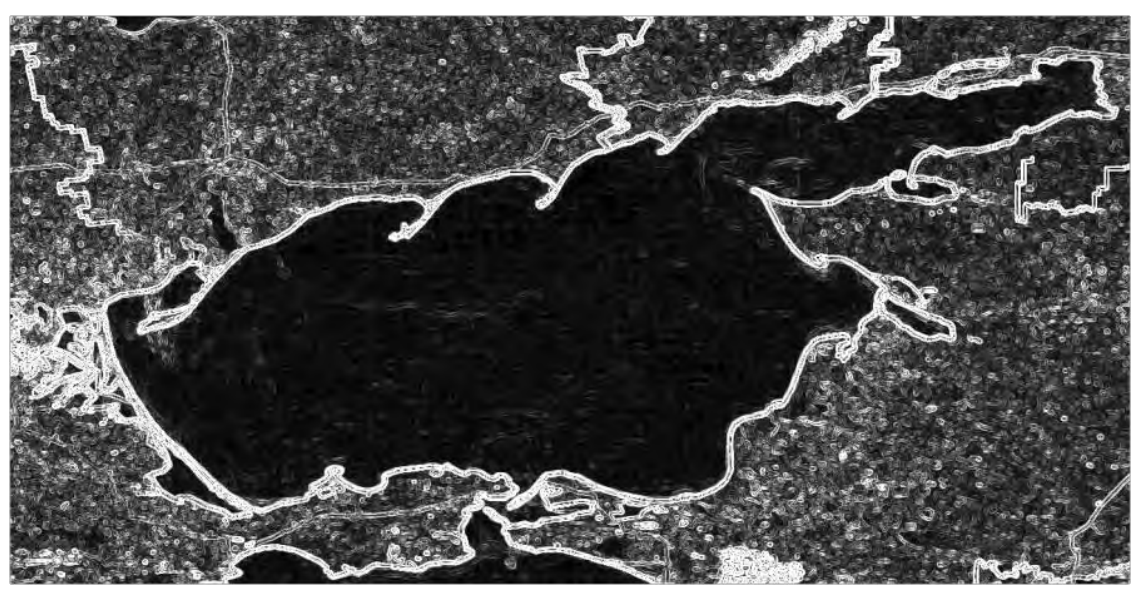

Fig. 4. Identification of contours by the Sobel method 
The next method to be considered is the Kirsch method. This one was developed by Russell A. Kirsch in 1971 [18]. This algorithm is based on the use of a detecting matrix, which is sequentially rotated along eight main cardinal directions: north, northwest, west, southwest, south, southeast, east and northeast [19]:

$$
\begin{aligned}
& E=\left[\begin{array}{ccc}
\overbrace{-3} & k_{0} & 5 \\
-3 & 0 & 5 \\
-3 & -3 & 5
\end{array}\right], \quad N E=\left[\begin{array}{ccc}
\overbrace{-3} & 5 & 5 \\
-3 & 0 & 5 \\
-3 & -3 & -3
\end{array}\right], \quad N=\left[\begin{array}{ccc}
\overbrace{5} & 5 & 5 \\
-3 & 0 & -3 \\
-3 & -3 & -3
\end{array}\right], \\
& N W=\left[\begin{array}{ccc}
\overbrace{5} & 5 & -3 \\
5 & 0 & -3 \\
-3 & -3 & -3
\end{array}\right], W=\left[\begin{array}{ccc}
k_{5} & -3 & -3 \\
5 & 0 & -3 \\
5 & -3 & -3
\end{array}\right], \quad S W=\left[\begin{array}{ccc}
c_{-3} & k_{5} & -3 \\
5 & 0 & -3 \\
5 & 5 & -3
\end{array}\right] \text {, } \\
& S=\left[\begin{array}{ccc}
\overbrace{-3} & k_{6} & -3 \\
-3 & 0 & -3 \\
5 & 5 & 5
\end{array}\right], S E=\left[\begin{array}{ccc}
\overbrace{-3} & -3 & 5 \\
-3 & 0 & 5 \\
-3 & 5 & 5
\end{array}\right]
\end{aligned}
$$

In this case, the boundary value is defined as the maximum value that can be found using the matrix, helping to obtain the maximum value. Here it can be concluded that $\mathrm{k}_{0}$ allows selecting vertical boundaries, and $\mathrm{k}_{5}$ - diagonal ones. It is also noticeable that the latter are similar to the first four ones, but are their mirror image along the central axis of the used matrix $[18,20]$.

As a result, we get Figure 5.

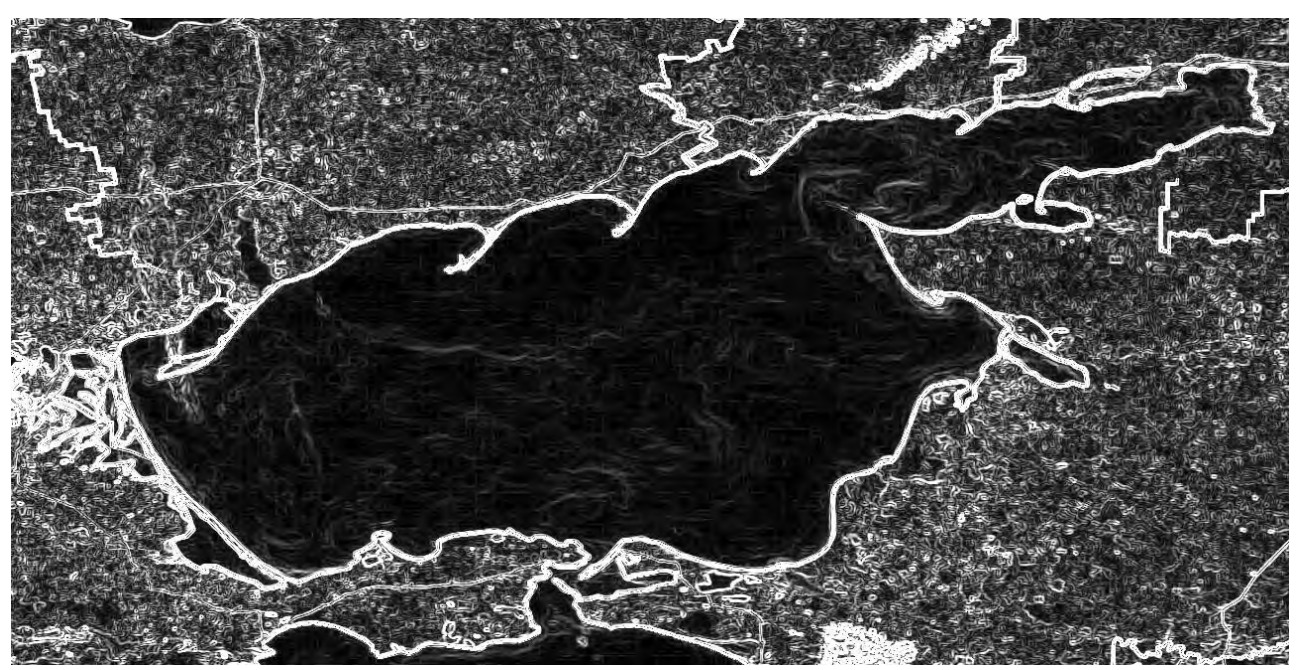

Fig. 5. Identification of contours using the Kirsch operator

The Laplace Gauss (LoG - Laplacian of Gaussian) operator was proposed in 1982 and is based on the calculation of symmetric circular derivatives $[18,19]$. This algorithm is defined as follows: 


$$
\nabla^{2} f=\frac{\partial^{2} f}{\partial^{2} x^{2}}+\frac{\partial^{2} f}{\partial^{2} y^{2}}
$$

The implementation includes two steps. The first one is smoothing the image with Gauss filter and after that calculating the Laplace function, which results in the formation of double contours. The determination is performed through finding zeros at the intersection of double boundaries. The LoG function is usually implemented through the following matrices:

$$
G_{x}=\left[\begin{array}{ccc}
0 & -1 & 0 \\
-1 & 4 & -1 \\
0 & -1 & 0
\end{array}\right], G_{y}=\left[\begin{array}{ccc}
-1 & -1 & -1 \\
-1 & 8 & -1 \\
-1 & -1 & -1
\end{array}\right]
$$

The result of this algorithm operation will be as follows (Fig. 6)

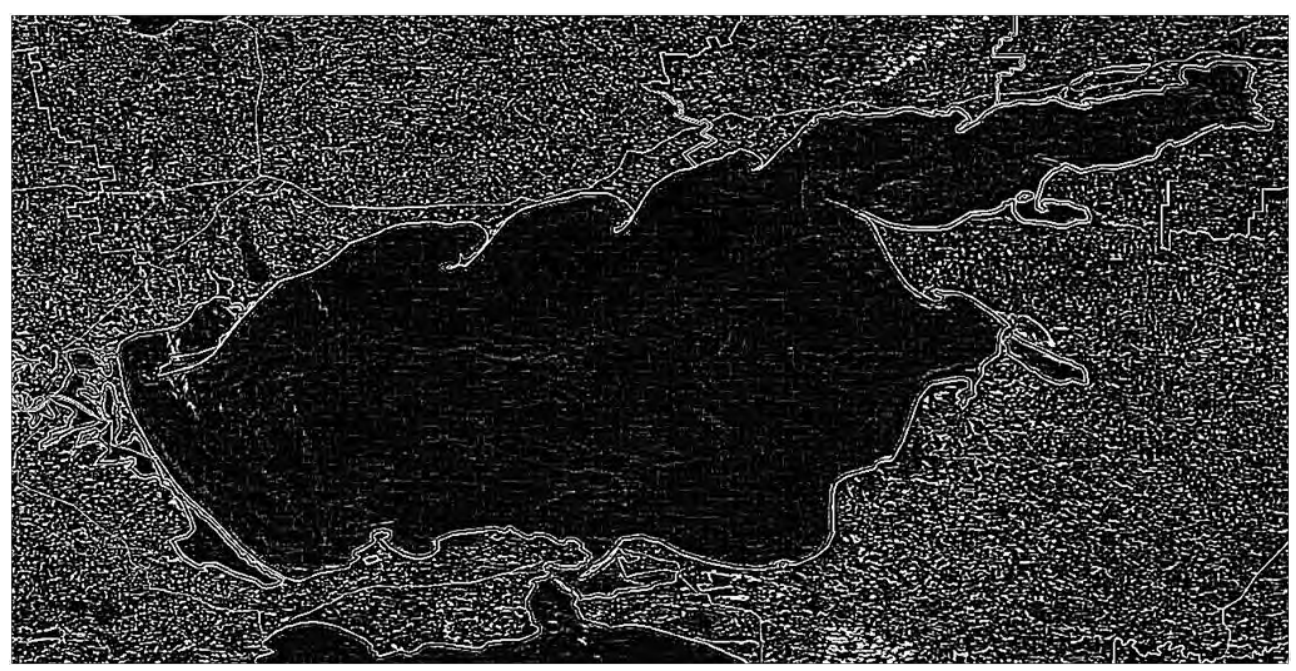

Fig. 6. Identification of contours using the LoG operator

As the final method for detecting boundaries, let us consider the Local Binary Patterns or LBP operator, which was first proposed in 1996 [21,24,22,23]. The essence of the operator is the application of threshold transformation to image pixels, in which the brightness value of the processed pixel is compared with the brightness values of pixels in its vicinity. The comparison results are converted to a binary value. In the basic case, a circular neighborhood with a radius of one pixel is used:

$$
\left[\begin{array}{lll}
5 & 9 & 1 \\
4 & 4 & 6 \\
7 & 2 & 3
\end{array}\right] \rightarrow\left[\begin{array}{lll}
1 & 1 & 0 \\
1 & & 1 \\
1 & 0 & 0
\end{array}\right]
$$

After applying the operator, the image is divided into rectangular parts, for each of which a histogram is calculated, showing how often pixels with different brightness are found. The brightness value of each pixel can be calculated using the following formula:

$$
\begin{array}{r}
\operatorname{LBP}(X, Y, W, H) \stackrel{\text { def }}{=} 2^{7} \cdot\left[s_{00} \geq s_{11}\right]+2^{6} \cdot\left[s_{10} \geq s_{11}\right]+2^{5} \cdot\left[s_{01} \geq s_{11}\right]+2^{0} \\
{\left[s_{01} \geq s_{11}\right]+2^{4} \cdot\left[s_{21} \geq s_{11}\right]+2^{1} \cdot\left[s_{02} \geq s_{11}\right]+2^{2} \cdot\left[s_{12} \geq s_{11}\right]+2^{3} \cdot\left[s_{22} \geq s_{11}\right]}
\end{array}
$$


Here:

$(X, Y)$ are coordinates of the upper left pixel of the considered area;

$(W, H)=(3 w, 3 h)$ is the size of the area to be calculated;

$s_{i, j} \stackrel{\text { def }}{=} \sum_{x=0}^{w-1} \sum_{y=0}^{h-1} p(X+i w+x, Y+j h+y)$ is the sum of pixels over the rectangle;

$p(x, y)$ is the pixel value of the image;

$[a \geq b] \stackrel{\text { def }}{=}\left\{\begin{array}{c}1, \text { если } a \geq b \\ 0, \text { иначе. }\end{array}[23,24]\right.$

An example of implementation is shown in Figure 7.

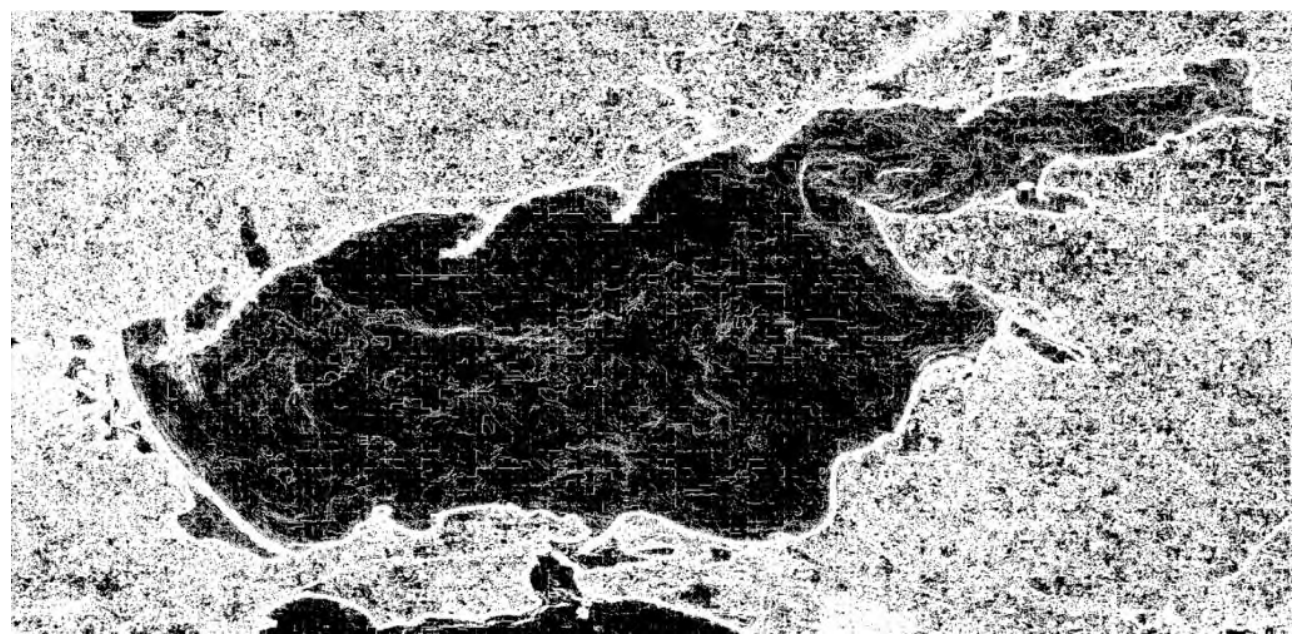

Fig. 7. Identification of contours by the LBP operator

\section{Conclusion}

In this article, an attempt was made for the practical study of methods for accentuation of contours of objects based on discontinuities in the brightness of a halftone image. The study of the relative performance of each of the methods presented in this article was carried.

The results of the work indicate the clarity and relevance of the use of high-tech methods for filtering ERS images. The modern methods of accentuation of contours of objects based on discontinuities in the brightness of a halftone image, used in real time or time close to it, have been considered, and their algorithms have been briefly described. Exploring each of the methods mentioned in this article is done using $\mathrm{C \#}$ software.

Based on the study, we can conclude that the LBP method can be effectively used for recognizing objects consisting of curved contours, which allows achieving the greatest sharpness of boundaries and high particularization. Although under other conditions, another method may turn out to be better. In further work, it is planned to improve the software implementation.

The reported study was funded by RFBR, project number 19-37-90070.

\section{References}

1. A. J. Lotka, Contribution to the energetics of evolution, Proc. Natl. Acad. Sci. 8, 147150 (1922) DOI:10.1073/pnas.8.6.147 
2. V. Volterra, Variations and fluctuations of the number of individuals in animal species living together, Rapp. P. - V. Reun. Cons. Int. Explor. Mer. 3, 3-51 (1928) DOI:10.1093/icesjms/3.1.3

3. G. I. Marchuk, A. S. Sarkisyan Mathematical modeling of ocean circulation, 304 (Moscow, Nauka, 1988)

4. R. V. Ozmidov, Diffusion of impurities in the ocean, 278, (Leningrad, Hydrometeoizdat, 1986)

5. G. F. Gause, Experimental studies on the struggle for existence: 1. Mixed population of two species of yeast, Journal of Experimental Biology, 9, 389-402 (1932)

6. E. A. Mitscherlich, Das Gesert des Minimums und das Gesetz des abnehmenden Bodenertrags, 595 (Landw, Jahrb, 1909)

7. G. G. Vinberg, Some results of practical application of production-hydrobiological methods, Production of populations and communities of aquatic organisms and methods of its study. Sverdlovsk: Ural center of the USSR Academy of Sciences, 1318 (1985)

8. I. I. Vorovich, A. S. Gorelov, A. B. Gorstko, Yu. A. Dombrovsky, Yu. A. Zhdanov, F. A. Surkov, L. V. Epstein, Rational use of water resources of the Azov sea basin: mathematical models, 360 (Moscow, Nauka, 1981)

9. H. T. Odum, System Ecology, 644 (New York, Wiley, 1983)

10. A. I. Abakumov, Signs of stability of water ecosystems in mathematical models, Proceedings Of the Institute of system analysis of the Russian Academy of Sciences. System analysis of the problem of sustainable development, 54, 49-60 (Moscow, ISA RAS, 2010)

11. S. E. Jorgensen, H. Mejer, M. Firiis, Examination of a lake model, Ecological Modelling, 4, 253-278 (1978)

12. N. D. Panasenko, A. I. Sukhinov, Using multichannel satellite images for recognition of " bloom" processes in shallow water on the example of the Azov Sea, Topical issues and innovative technologies in the development of geographical sciences collection of works of the All-Russian Scientific Conference, 615-618 (2020)

13. V. B. Kashnin, A. I. Sukhinin, Remote sensing of the Earth from space. Digital Image Processing: A Tutorial, 264 (Moscow, Logos, 2001)

14. A. L. Leontyev, A. V. Nikitina, M. I. Chumak, Application of assimilation and filtration methods for satellite water sensing data for plankton population evolution processes predictive modelling, Computational Mathematics and Information Technologies, 1(1), 1-11 (2020)

15. O. Yu. Lavrova, D. M. Soloviev, A. Ya. Strochkov, V. D. Shendrik, Satellite monitoring of intense algal blooms in the Rybinsk reservoir, Modern problems of remote sensing of the Earth from space, 11(3), 54-72 (2014)

16. The official website of NASA Worldview, http://worldview.earthdata.nasa.gov (Last accessed 24.06.2020)

17. The official website of Roscosmos Geoportal, https://www.gptl.ru (Last accessed 24.06.2020)

18. R. S. Gonzalez, R. E. Woodsue, Digital image processing, 1081 (Moscow, Technosphere, 2012)

19. U. Pret, Digital image processing, 480 (Moscow, Mir, 1982) 
20. R. Kirsch, Computer determination of the constituent structure of biological images, Computers and Biomedical Research, 4(3), 315-328 (1971) DOI: 10.1016 / 00104809 (71) 90034-6

21. T. Maenpaa, The local binary pattern approach to texture analysis - Extensions and Applications (Oulu University Press, 2003)

22. V. I. Petruk, A. V. Samorodov, I. N. Spiridonov, Application of local binary patterns to solving the problem of face recognition. Bulletin of the Moscow State Technical University. N.E. Bauman. Series: Instrumentation, 58-63 (2011)

23. A. A. Sukhinov, G. B. Ostrobrod, Efficient Face Detection on Epiphany Multicore Processor. Computational Mathematics and Information Technologies. Electronic journal, 1(1), 113-127 (2017)

24. N. D. Panasenko, M. Ganzhur, N. Dyachenko, O. Smirnova, A. Poluyan, Recognition of "flowering" processes on the base of remote sensing data in shallow water ponds on the example of the Azov Sea, E3S Web of Conferences, 175, 12013 (2020) 